\title{
Unexpected long-term complications of atrial septal defects closure
}

\author{
Piotr Weryński ${ }^{1}$, Robert Sabiniewicz ${ }^{2}$ \\ ${ }^{1}$ Department of Pediatric Cardiology, Jagiellonian University Medical College, Krakow, Poland \\ ${ }^{2}$ Department of Pediatric Cardiology and Congenital Heart Disease, Medical University of Gdansk, Gdansk, Poland
}

Adv Interv Cardiol 2020; 16, 4 (62): 514-515

DOI: https://doi.org/10.5114/aic.2020.101782

Percutaneous closure of ostium secundum atrial septal defects (ASD II) is the contemporary treatment of choice. It has proven to be effective and safe, with very low complication risk. We present two patients: a 9-yearold boy with an 18-mm-diameter ASD II closed using the 20-mm Cardia Ultrasept occluder and a 6-year-old girl with a 16-mm-diameter ASD II closed using the 20-mm Ultrasept occluder without any periprocedural complications. The positioning of the devices was correct and complete closure of the shunts was confirmed in the follow-up echocardiography. Patients were in routine follow-up and echocardiographic examination 4 and 3 years after implantation respectively. The follow-up revealed a few streams of left-to-right shunts through the device. The shunts were hemodynamically significant, causing volume overload and right atrial and right ventricular enlargement. Consequently, the devices were surgically removed and the ASDs were closed with pericardial patches 6 and 4.5 years after implantation respectively. Visual inspection revealed multiple perforations in the left and right parts of both devices - only nitinol struts with small parts of the devices were covered with endothelium (Figures 1 A, B). The Ultrasept occluder is intended for closure of interatrial communications. It consists of two discs connected with a self-centering waist made of a nitinol wire frame and covered with an Ivalon (polyvinyl alcohol - PVA) membrane (Figures 1 C, D). PVA is a synthetic polymer commonly used in medical devices due to its biocompatibility and high water solubility [1]. The Ultrasept occluder is a well-proven implant with good midterm results. In our patients, complete closure of the ASDs was observed in follow-up echocardiography 3 and 4 years after implantation with the next examination revealing significant shunts. It was probably due to incomplete endothelization of the device prior to the material's absorption by the tissue. Similar dissolution of the PVA membrane has previously been reported as early as 1 week and as late as 2 years after implantation. Both options for surgical removal of the damaged device and surgical closure of the defect as well as percutaneous closure of leaks with the new device were used [2, 3]. Also, the nitinol wire frame fracture was observed in the similar Cardia Ultrasept device. Because of the risk of late or very late complications after percutaneous ASD closure patients require regular and life-long follow-up care. Any complication related to the device should be reported and analyzed to facilitate implant modification and constructional changes. The Cardia Ultrasept II with interposed Gore-Tex patch is the modified last generation of Cardia devices. The new device was demonstrated to be safe and feasible. With the interposition of the Gore-Tex, it has been possible to abolish perforation of the Ivalon membrane as a complication. Such conclusions can be drawn from a study on 30 Mexican patients after atrial septal defect closure with no incidence of perforation at follow-up for 6 (range: 1-15) months [4].

\section{Conflict of interest}

The authors declare no conflict of interest.

\section{Corresponding author:}

Prof. Robert Sabiniewicz, Department of Pediatric Cardiology and Congenital Heart Disease, Medical University of Gdansk, Gdansk, Poland, phone: +48 695687 587, e-mail: sabini@gumed.edu.pl

Received: 12.05.2020, accepted: 12.09.2020. 

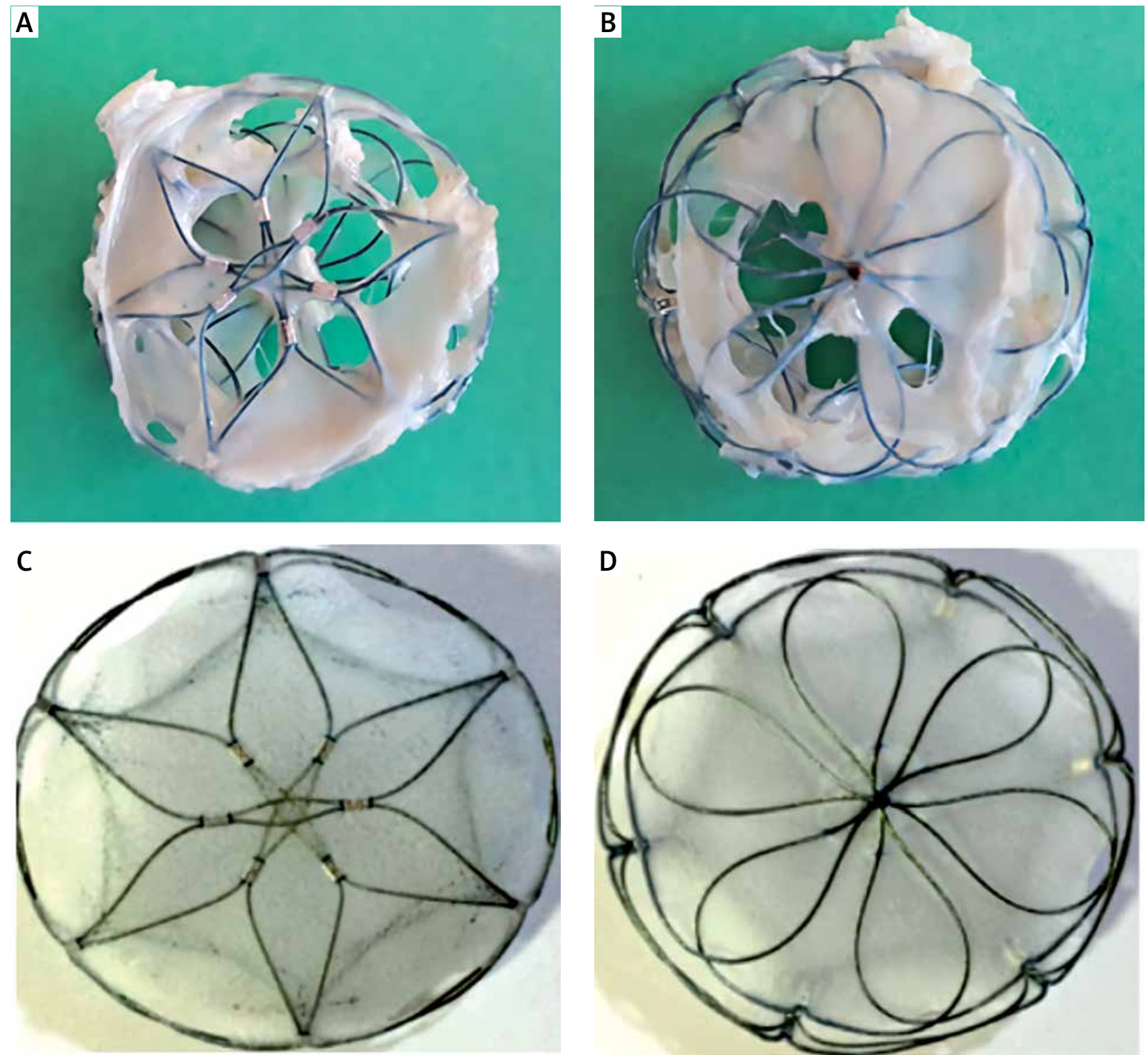

Figure 1. Cardia Ultrasept occluder, the removed device as seen from the left atrial side (A) and right atrial side (B). Cardia Ultrasept occluder as seen from the left atrial side (C) and right atrial side (D)

\section{References}

1. Alexandre N, Ribeiro J, Gärtner A, et al. Biocompatibility and hemocompatibility of polyvinyl alcohol hydrogel used for vascular grafting: in vitro and in vivo studies. J Biomed Mater Res A 2014; 102: 4262-75.

2. Bartel T, Bonaros N, Muller S. Device failure weeks to months after transcatheter closure of secundum type atrial septal defects. Heart 2010; 96: 1603.

3. Bozyel S, Sahin T, Dervis E, at al. A massive left-to-right shunt due to delayed spontaneous perforation of polyvinyl alcohol membrane of atrial septal occluder. Turk Kardiyol Dern Ars 2017; 45: 541-4.

4. Mijangos-Vázquez R, García-Montes AJ, Soto-López EM, et al. Atrial septal defect closure with the new Cardia Ultrasept IIM $^{\mathrm{TM}}$ device with interposed Goretex patch: Mexican experience has the perforation of Ivalon's membrane been solved? Cardiol Young 2018; 28: 709-14. 